\title{
Modelagem morfométrica de Licania tomentosa (Benth.) por regressão logística e máquinas vetor de suporte
}

\author{
Morphometric modeling of Licania tomentosa (Benth.) by logistic regression and support- \\ vector machines
}

\author{
B. O. Lafetá*; F. F. da Silva; M. A. dos Santos; I. A. Pimenta; I. C. I. Fontan; N. \\ R. Fonseca; C. J. Sartori \\ Departamento de Engenharia Florestal, Instituto Federal de Educação, Ciência e Tecnologia de Minas Gerais, \\ 39705-000, São João Evangelista-MG, Brasil
}

*bruno.lafeta@ifmg.edu.br

(Recebido em 21 de abril de 2020; aceito em 16 de junho de 2020)

\begin{abstract}
Investigações científicas sobre a morfometria de árvores subsidiam o planejamento e gerenciamento racionais da arborização urbana, importante para a sustentabilidade ambiental de cidades. O objetivo foi avaliar a eficiência da modelagem de relações morfométricas interdimensionais da copa de L. tomentosa empregando regressão logística e Máquinas Vetor de Suporte (MVS), identificando potenciais usos no planejamento da arborização urbana. O inventário foi conduzido nos municípios de São João Evangelista e Peçanha em Minas Gerais. Foram amostradas 116 árvores em 21 vias públicas. Calcularam-se o formal de copa, índice de saliência, índice de abrangência, grau de esbeltez, proporção de copa e área de projeção de copa. Foram testados dois métodos de modelagem para a estimativa de altura total, altura de inserção da copa e índices morfométricos de copa $(r, p \leq 0,01)$ em função exclusivamente do DAP, uma por regressão não linear (modelo logístico) e outra por MVS. A altura total, altura de inserção da copa, formal de copa, índice de abrangência e área de projeção de copa aumentaram à medida que fustes se tornaram mais grossos. O decrescimento com o aumento do DAP foi observado somente para o grau de esbeltez. Relações morfométricas podem ser estimadas com precisão por modelos de regressão e MVS. Informações da média assintótica do estoque de crescimento de L. tomentosa em altura total, altura de inserção da copa, área de projeção da copa e raio de copa são úteis para a definição do local de seu plantio em áreas urbanas, indicado para praças, canteiros e/ou calçadões.

Palavras-chave: arborização urbana, inteligência computacional, regressão não linear.
\end{abstract}

Scientific investigations on the tree morphometry support rational planning and management of urban afforestation, important for environmental sustainability of cities. This work aimed to evaluate efficiency modeling of interdimensional morphometric relationships of $L$. tomentosa crown and stem using logistic regression and Support-Vector Machines (MVS), identifying potential uses in the planning of urban afforestation. Inventory was conducted in the São João Evangelista and Peçanha municipalities in Minas Gerais. 116 trees were sampled on 21 public roads. The crown formal, salience index, range index, slenderness rate, crown proportion and crown projection area were calculated. Two modeling methods were tested to estimate total height, crown insertion height and crown morphometric indices $(r, p \leq 0.01)$ in function exclusively of DBH, one by non-linear regression (logistic model) and the other by MVS. Total height, crown insertion height, formal crown, range index and crown projection area increased as the stem became thicker. Decrease with the increase in DAP was observed only for slenderness rate. Regression models and MVS can estimate morphometric relationships accurately. Information on the asymptotic average of the growth stock of $L$. tomentosa at total height, crown insertion height, crown projection and crown radius are useful for defining your planting location in urban areas, suitable for squares, flowerbeds and/or boardwalks.

Keywords: urban afforestation, computational intelligence, nonlinear regression.

\section{INTRODUÇÃO}

A análise do comportamento morfométrico da copa e fuste de árvores é fundamental para melhor aproveitamento do espaço verde em cidades [1]. Espaços verdes têm sido frequentemente planejados a partir de manuais, que padronizam práticas silviculturais e desconsideram a influência da qualidade de sítio e fatores genéticos. 
A arborização promove serviços sociais e ambientais para o bem-estar humano, como melhorias estéticas, sombreamento, direcionamento de vento, redução de poluições atmosférica, sonora e visual, estímulo a atividades recreativas, alimento e abrigo para a fauna, conectividade entre paisagens e atenuação de ilhas de calor $[2,3]$. Todavia, a arborização de cidades é uma prática que requer eficiente planejamento e gerenciamento para se evitar prejuízos advindos do crescimento não monitorado de árvores, como danos em calçadas, tubulações subterrâneas, edifícios e redes elétricas [4]. A escolha de espécies deve considerar caraterísticas de crescimento, paisagísticas e inerentes ao local de plantio, como a disponibilidade de espaço.

Licania tomentosa (Benth.) (Chrysobalanaceae) por sua vez, também conhecida por oiti ou oitizeiro, ocorre naturalmente desde Pernambuco até o Norte do Espírito Santo e Vale do Rio Doce do estado de Minas Gerais, estando associada à Mata Atlântica [5]. Esta espécie é ornamental e amplamente difundida na arborização urbana no Brasil, podendo ser utilizada em planos para recuperação de áreas degradadas [6]. Os frutos são comestíveis e explorados para a produção em pequena escala de compotas, geleias e farinhas [6, 7]. Entretanto, ainda, são poucas pesquisas sobre a morfometria de árvores dessa espécie, sobretudo, em ambiente urbano.

A morfometria de árvores se modifica naturalmente em virtude do genótipo, fisiografia, tempo, prescrições silviculturais e condições edáficas e climáticas $[8,9,10]$. Relações interdimensionais permitem a análise de mudanças morfométricas, reconstrução do espaço ocupado por árvore e inferências sobre a produtividade, estabilidade e serviços ambientais [2, 11]. No entanto, o levantamento de muitos dados biométricos pode inviabilizar operacionalmente inventários florestais, uma vez que medições da copa consomem muito tempo e são laboriosas. A redução desse esforço amostral pode ser realidade com o emprego de técnicas de modelagem.

$\mathrm{O}$ avanço de recursos computacionais tem propiciado o uso de modelos de regressão não lineares e inteligência computacional. A modelagem permite a compreensão indireta de como interações e processos ecológicos complexos atuam no crescimento de árvores isoladas ou arvoredos, estabelecendo relações funcionais entre aspectos morfométricos e preditores de fácil e rápida medição, como a circunferência ou diâmetro de fustes [4]. Curvas sigmoidais (forma em S) subsidiam o estabelecimento de prescrições silviculturais e a avaliação de capacidade produtiva, que pode ser expressa em termos de altura, área de projeção da copa, diâmetro da copa e/ou volume [12, 13, 14].

Modelos de regressão não lineares de característica sigmoidal são rotineiramente empregados no setor florestal $[1,12,15,16,17]$. No caso específico do modelo logístico, a fundamentação biológica e a facilidade de interpretação de parâmetros propiciam uma rápida análise de confiabilidade das estimativas quanto a convergência do algoritmo de parametrização. Trata-se de um modelo cuja forma original apresenta três parâmetros, assíntota horizontal e pontos de inflexão e escala [16, 18]. Por outro lado, algoritmos de aprendizagem de máquina têm sido cada vez mais utilizados para a modelagem de dados biológicos. O método de Máquinas Vetor de Suporte (MVS), desenvolvido por Vapnik (1995) [19], é uma abordagem de aprendizado supervisionado capaz solucionar problemas relacionados a classificação de padrões, regressão e detecção de outliers [20]. Diferencia das redes neurais artificiais face ao princípio de minimização de risco estrutural, que sempre converge para um ótimo global [21].

A aplicação de diferentes métodos estatísticos para estimativas biométricas de árvores se relaciona ao fato de que pequenas melhorias em exatidão representam ganhos significativos na qualidade da tomada de decisões por gestores florestais. Investigações científicas sobre a modelagem do comportamento morfométrico de árvores em áreas urbanas são escassas e a falta de informações pode ser justificada pela carência de políticas públicas e planos diretores que estimulem o plantio de árvores em vias públicas sob orientação técnica. Mediante o exposto, o objetivo do presente trabalho foi avaliar a eficiência da modelagem de relações morfométricas interdimensionais da copa e fuste de L. tomentosa empregando regressão logística e MVS, identificando potenciais usos no planejamento da arborização urbana. 


\section{MATERIAL E MÉTODOS}

\subsection{Caracterização experimental}

O trabalho foi conduzido nos municípios de São João Evangelista (altitude de 700m) e Peçanha (altitude de 690m), inseridos no Vale do Rio Doce em Minas Gerais, Brasil. A distância linear entre os municípios é de $22 \mathrm{Km}$. O clima da região é do tipo Cwa (temperado chuvosomesotérmico) pela classificação do sistema internacional de Köppen, com verão chuvoso e inverno seco. As médias anuais de temperatura e precipitação em São João Evangelista são de $20,2^{\circ} \mathrm{C}$ (médias variando de $16,7^{\circ} \mathrm{C}$ em julho a $22,8^{\circ} \mathrm{C}$ em janeiro e fevereiro) e $1.377 \mathrm{~mm}$ (médias variando de $10 \mathrm{~mm}$ em julho e agosto a $308 \mathrm{~mm}$ em dezembro), respectivamente. Nesta sequência, Peçanha apresenta média de $20,4^{\circ} \mathrm{C}$ (médias variando de $17,3^{\circ} \mathrm{C}$ em julho a $23,1^{\circ} \mathrm{C}$ em janeiro) e $1.339 \mathrm{~mm}$ (médias variando de $13 \mathrm{~mm}$ em agosto a $287 \mathrm{~mm}$ em dezembro). Os dados climáticos foram oriundos de registros anuais de 1982 a 2012, disponibilizados gratuitamente por Climatedata.org (http://es.climate-data.org/).

Foram amostradas 116 árvores da espécie L. tomentosa distribuídas em 21 vias públicas, 6 vias em São João Evangelista e o restante, em Peçanha. As árvores se encontravam sem sinais aparentes de poda, injúrias ou ataque de insetos. Mensurou-se a circunferência a 1,30 $\mathrm{m}$ de altura do solo (Circunferência a Altura do Peito, CAP, $\mathrm{cm}$ ) de todos os fustes empregando fita métrica; o diâmetro equivalente foi calculado para as árvores com tronco bifurcado abaixo do ponto de medição. A altura total $(\mathrm{H}, \mathrm{m})$ e altura de inserção da copa (HIC - altura do primeiro galho vivo, caracterizado pela presença de folhas, $\mathrm{m}$ ) foram tomadas com auxílio de vara telescópica. $\mathrm{O}$ comprimento da copa $(\mathrm{L}, \mathrm{m})$ foi calculado pela diferença entre H e HIC e o diâmetro da copa (Dc, m), pela média dos diâmetros da copa, obtida da medição de quatro raios nas direções paralela e perpendicular à orientação das vias públicas através da projeção vertical da copa.

Os seguintes índices morfométricos baseados no tamanho e na morfologia da árvore, conforme Sterba (1991) [11] e Durlo e Denardi (1998) [22], foram avaliados: Formal de Copa (FC), Índice de Saliência (IS), Índice de Abrangência (IA), Grau de Esbeltez (GE), Proporção de Copa (PC) e Área de Projeção de Copa $\left(\mathrm{APC}, \mathrm{m}^{2}\right)$. Os índices foram assim expressos: $F C=D c / L ; I S=$ $D c / D A P ; I A=D c / H ; G E=H / D A P ; P C=100 L / H$ e $A P C=D C^{2} \cdot \pi / 4$.

Os dados foram divididos aleatoriamente em grupos de parametrização (70\% das amostras) e validação (30\% das amostras), mutualmente exclusivos (técnica holdout), adotando método randômico de amostragem sem reposição. Nessa divisão, árvores de ambos os municípios estavam inclusas nas amostras. Os dados de altura (H e HIC) e índices morfométricos (FC, IS, IA, GE, PC e APC) foram submetidos à análise de correlação de Pearson (r) com o DAP. A fim de minimizar problemas com a perda de precisão de preditores, assumiu-se a ausência de significância estatística de correlação pelo teste t $(r, p>0,01)$ como critério para a exclusão de variáveis durante a fase de parametrização.

\subsection{Métodos de modelagem e avaliação}

Foram testados dois métodos de modelagem para a estimativa de alturas e índices morfométricos de copa $(\mathrm{r}, \mathrm{p} \leq 0,01)$ em função exclusivamente do DAP, uma por regressão não linear e outra por MVS. Optou-se pelo DAP como variável preditora por ser facilmente obtida e convencionalmente analisada em inventários florestais. A análise de regressão foi realizada através do método iterativo de Levenberg-Marquardt, com o ajuste do modelo logístico de três parâmetros, $Y=\alpha\left(1+\beta e^{-\gamma X}\right)^{-1}$; em que $\alpha, \beta$ e $\gamma$ são parâmetros do modelo logístico.

A construção de máquinas de vetores se baseou no processo de aprendizado estatístico descrito em detalhes por Vapnik (1995) [19] e Meyer et al. (2017) [23]. As MVS foram implementadas usando o pacote R "e1071" [23] com parâmetro de custo igual a 1, gama de 0,1 e épsilon de 0,1; parâmetros definidos por análise exploratória, com valores oscilando entre 0,001 e 10. Para a parametrização das MVS, foram analisados quatro tipos de funções Kernel (linear, base radial, sigmoide e polinomial). As funções Kernel são algoritmos que melhoram o poder computacional das máquinas de aprendizagem, possibilitando representar fenômenos de maior complexidade. 
Somente um tipo de função Kernel foi selecionada para compor as MVS nas análises gráficas subsequentes.

As seleções do método de modelagem e da função Kernel para compor as MVS para as estimativas de altura e índices morfométricos de copa se basearam na Média dos Desvios Absolutos (MDA), Raiz Quadrada do Erro Médio (RQEM), e coeficiente de correlação (r) entre valores observados e estimados. As análises gráficas consistiram na inspeção estatística da dispersão dos resíduos padronizados e dos valores observados em relação àqueles estimados.

Os pontos que extrapolaram a tendência geral dos dados não foram eliminados das análises estatísticas a fim de se verificar a capacidade da regressão logística e SVM em lidar com outliers ou ruídos. Para diagnóstico de efeito estatístico, empregou-se 1\% de significância em todas as análises. Estas foram efetuadas com auxílio dos softwares Curve Expert 1.4 e R versão 3.5.2 [24].

\section{RESULTADOS}

No grupo de parametrização, as árvores de menor e maior DAP apresentaram 23 e $74 \mathrm{~cm}$ em São João Evangelista e 4 e $41 \mathrm{~cm}$ em Peçanha, respectivamente. Na validação, as variações de DAP foram de 28 a $61 \mathrm{~cm}$ no primeiro município e de 9 a $55 \mathrm{~cm}$ no segundo.

$\mathrm{Na}$ fase de parametrização, a correlação positiva significativa $(\mathrm{p} \leq 0,01)$ do DAP foi evidenciada com a altura $(\mathrm{r}=0,8733)$, altura do primeiro galho vivo $(\mathrm{r}=0,8733)$, formal de copa $(\mathrm{r}=0,3550)$, índice de abrangência $(\mathrm{r}=0,7269)$ e área de projeção da copa $(\mathrm{r}=0,8973)$. Somente o grau de esbeltez exibiu correlação negativa significativa $(r=-0,6588, p \leq 0,01)$. Logo, selecionaram-se esses seis últimos atributos para as modelagens de relação interdimensional. As correlações do índice de saliência e proporção de copa tiveram módulos inferiores a $0,1(p>0,01)$.

As relações interdimensionais estabelecidas em função do DAP se destinaram à obtenção de estimativas de altura total, altura do primeiro galho vivo, formal de copa, índice de abrangência, grau de esbeltez e área de projeção da copa (Tabela 1). Como esperado, a assíntota, representada pelo parâmetro " $\alpha$ ", foi positiva em todos os ajustes realizados com o modelo logístico. A modelagem via regressão apresentou poucos desvios nas fases de parametrização e treinamento, com baixos valores de MDA e RQEM. A precisão das estimativas aumentou com o grau de associação entre variáveis resposta e preditoras.

Tabela 1: Coeficientes e qualidade de ajuste do modelo logístico para a estimativa de alturas e índices morfométricos da copa em função do DAP de árvores de L. tomentosa, da arborização urbana de São João Evangelista e Peçanha, em Minas Gerais, Brasil.

\begin{tabular}{|c|c|c|c|c|c|c|}
\hline Atributos & $\mathbf{H}$ & HIC & FC & IA & GE & APC \\
\hline$\alpha$ & 10,212522 & 4,479086 & 2,809875 & 1,459569 & 0,188298 & 130,274676 \\
\hline$\beta$ & 3,472545 & 4,271139 & 4,041158 & 2,480117 & $-0,988345$ & 86,138257 \\
\hline & 0,062083 & 0,114873 & 0,123407 & 0,049854 & 0,072739 & 0,108048 \\
\hline & \multicolumn{6}{|c|}{----------------- Parametrização ------------------ } \\
\hline MDA & 0,7202 & 0,8381 & 0,7028 & 0,1480 & 0,0341 & 8,5471 \\
\hline RQEM & 0,9063 & 1,0460 & 1,1204 & 0,1930 & 0,0459 & 13,7012 \\
\hline \multirow[t]{2}{*}{$\mathrm{r}$} & $0,8914^{* *}$ & $0,6406^{* *}$ & $0,4084^{* *}$ & $0,7387^{* *}$ & $0,8710^{* *}$ & $0,9200^{* *}$ \\
\hline & \multicolumn{6}{|c|}{--------------------- Validação --------------------- } \\
\hline MDA & 0,9827 & 1,0208 & 1,1341 & 0,2146 & 0,0379 & 14,2209 \\
\hline RQEM & 1,2998 & 1,2558 & 1,5993 & 0,2660 & 0,0477 & 23,1088 \\
\hline $\mathrm{r}$ & $0,7932^{* *}$ & $0,5326^{* *}$ & $0,5382^{* *}$ & $0,5052^{* *}$ & $0,7063^{* *}$ & $0,7805^{* *}$ \\
\hline
\end{tabular}

$\mathrm{H}=$ altura total; $\mathrm{HIC}=$ altura do primeiro galho vivo; $\mathrm{FC}=$ formal de copa; $\mathrm{IA}=$ índice de abrangência; $\mathrm{GE}=$ grau de esbeltez; APC = área de projeção de copa; $\alpha, \beta$ e $\gamma=$ parâmetros do modelo logístico; MDA = média dos desvios absolutos; RQEM = raiz quadrada do erro médio e; $r$ = coeficiente de correlação de Pearson. ${ }^{* *}$ significativo a $1 \%$ de probabilidade pelo teste $t$. 
Dentre as funções Kernel, as MVS de base radial tiveram melhor desempenho na estimativa de alturas e índices morfométricos de L. tomentosa nas fases de parametrização e validação (Tabela 2). Em termos gerais, no que se refere às estatísticas para avaliar a qualidade preditiva dos métodos de modelagem, a regressão se expressou melhor do que as MVS na fase de validação. Nesta fase de processamento, a correlação entre os valores observados e estimados para o formal de copa pela regressão foi 2,4 vezes maior que aquela via MVS.

Tabela 2: Estatísticas das aproximações por máquinas de vetores de suporte construídas para a estimativa de alturas e índices morfométricos da copa em função do DAP de árvores de L. tomentosa, da arborização urbana de São João Evangelista e Peçanha, em Minas Gerais, Brasil.

\begin{tabular}{|c|c|c|c|c|c|c|c|}
\hline Kernel & Atributos & $\mathbf{H}$ & HIC & FC & IA & GE & APC \\
\hline & & \multicolumn{6}{|c|}{------------------ Parametrização ------------------ } \\
\hline \multirow{3}{*}{ Linear } & MDA & 0,7800 & 0,8842 & 0,6866 & 0,1522 & 0,0402 & 11,2206 \\
\hline & RQEM & 0,9900 & 1,0857 & 1,1636 & 0,1984 & 0,0748 & 15,6543 \\
\hline & $\mathrm{r}$ & $0,8733^{* *}$ & $0,6070^{* *}$ & $0,3550^{* *}$ & $0,7269^{* *}$ & $0,6588^{* *}$ & $0,8973^{* *}$ \\
\hline \multirow{3}{*}{ Radial } & MDA & 0,7045 & 0,8631 & 0,6799 & 0,1495 & 0,0384 & 8,5115 \\
\hline & RQEM & 0,9188 & 1,0635 & 1,1587 & 0,1934 & 0,0708 & 13,8007 \\
\hline & $\mathrm{r}$ & $0,8901^{* *}$ & $0,6251^{* *}$ & $0,3947^{* *}$ & $0,7404^{* *}$ & $0,7086^{* *}$ & $0,9196^{* *}$ \\
\hline \multirow{3}{*}{ Sigmoide } & MDA & 0,7990 & 0,8912 & 0,6877 & 0,1534 & 0,0405 & 11,3654 \\
\hline & RQEM & 0,9926 & 1,0847 & 1,1640 & 0,2025 & 0,0749 & 16,5234 \\
\hline & $\mathrm{r}$ & $0,8692^{* *}$ & $0,6065^{* *}$ & $0,3574^{* *}$ & $0,7229^{* *}$ & $0,6548^{* *}$ & $0,8970^{* *}$ \\
\hline \multirow{3}{*}{ Polinomial } & MDA & 1,3764 & 1,0473 & 0,7305 & 0,2023 & 0,0531 & 21,0932 \\
\hline & RQEM & 1,8486 & 1,2763 & 1,2171 & 0,2626 & 0,0903 & 29,9144 \\
\hline & $\mathrm{r}$ & $0,5284^{* *}$ & $0,4053^{* *}$ & $0,2108^{\mathrm{ns}}$ & $0,4431^{* *}$ & $0,4327^{* *}$ & $0,6537^{* *}$ \\
\hline \multicolumn{8}{|c|}{ 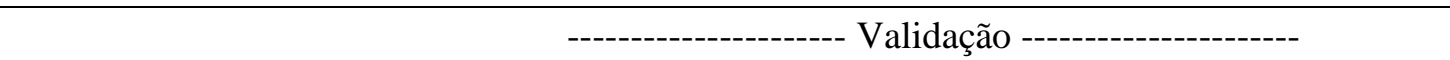 } \\
\hline \multirow{3}{*}{ Linear } & MDA & 1,0167 & 1,1154 & 0,8250 & 0,2128 & 0,0339 & 13,8358 \\
\hline & RQEM & 1,3708 & 1,3570 & 1,9820 & 0,2650 & 0,0433 & 19,1133 \\
\hline & $\mathrm{r}$ & $0,7708^{* *}$ & $0,4447^{* *}$ & $0,1542^{\text {ns }}$ & $0,4951^{* *}$ & $0,7651^{* *}$ & $0,7950^{* *}$ \\
\hline \multirow{3}{*}{ Radial } & MDA & 0,9374 & 1,0673 & 0,8067 & 0,2115 & 0,0348 & 13,7848 \\
\hline & RQEM & 1,3218 & 1,3005 & 1,9658 & 0,2617 & 0,0427 & 21,9901 \\
\hline & $\mathrm{r}$ & $0,7894^{* *}$ & $0,4947^{* *}$ & $0,2268^{\mathrm{ns}}$ & $0,5039^{* *}$ & $0,7716^{* *}$ & $0,7845^{* *}$ \\
\hline \multirow{3}{*}{ Sigmoide } & MDA & 1,0414 & 1,1050 & 0,8203 & 0,2132 & 0,0335 & 12,8383 \\
\hline & RQEM & 1,3251 & 1,3295 & 1,9810 & 0,2657 & 0,0433 & 17,9268 \\
\hline & $\mathrm{r}$ & $0,7687^{* *}$ & $0,4436^{* *}$ & $0,1576^{\mathrm{ns}}$ & $0,4933^{* *}$ & $0,7632^{* *}$ & $0,7963^{* *}$ \\
\hline \multirow{3}{*}{ Polinomial } & MDA & 1,4813 & 1,1940 & 0,8208 & 0,2400 & 0,0451 & 17,5365 \\
\hline & RQEM & 1,6575 & 1,3874 & 2,0076 & 0,2908 & 0,0574 & 25,3893 \\
\hline & $\mathrm{r}$ & $0,5768^{* *}$ & $0,3186^{\mathrm{ns}}$ & $0,0417^{\mathrm{ns}}$ & $0,3710^{\mathrm{ns}}$ & $0,6294^{* *}$ & $0,6433^{* *}$ \\
\hline
\end{tabular}

$\mathrm{H}=$ altura total; $\mathrm{HIC}=$ altura do primeiro galho vivo; $\mathrm{FC}=$ formal de copa; $\mathrm{IA}=$ índice de abrangência; $\mathrm{GE}=$ grau de esbeltez; $\mathrm{APC}=$ área de projeção de copa; $\mathrm{MDA}=$ média dos desvios absolutos; $\mathrm{RQEM}=$ raiz quadrada do erro médio e; $\mathrm{r}=$ coeficiente de correlação de Pearson. ${ }^{* *, n s}$ significativo e não significativo a $1 \%$ de probabilidade pelo teste $t$, respectivamente.

Observou-se comportamento homocedástico dos resíduos padronizados em ambos os métodos de modelagem avaliados, exceto nas ocasiões em que se avaliaram a altura do primeiro galho vivo, grau de esbeltez e a área de projeção da copa (Figura 1). 
Logístico
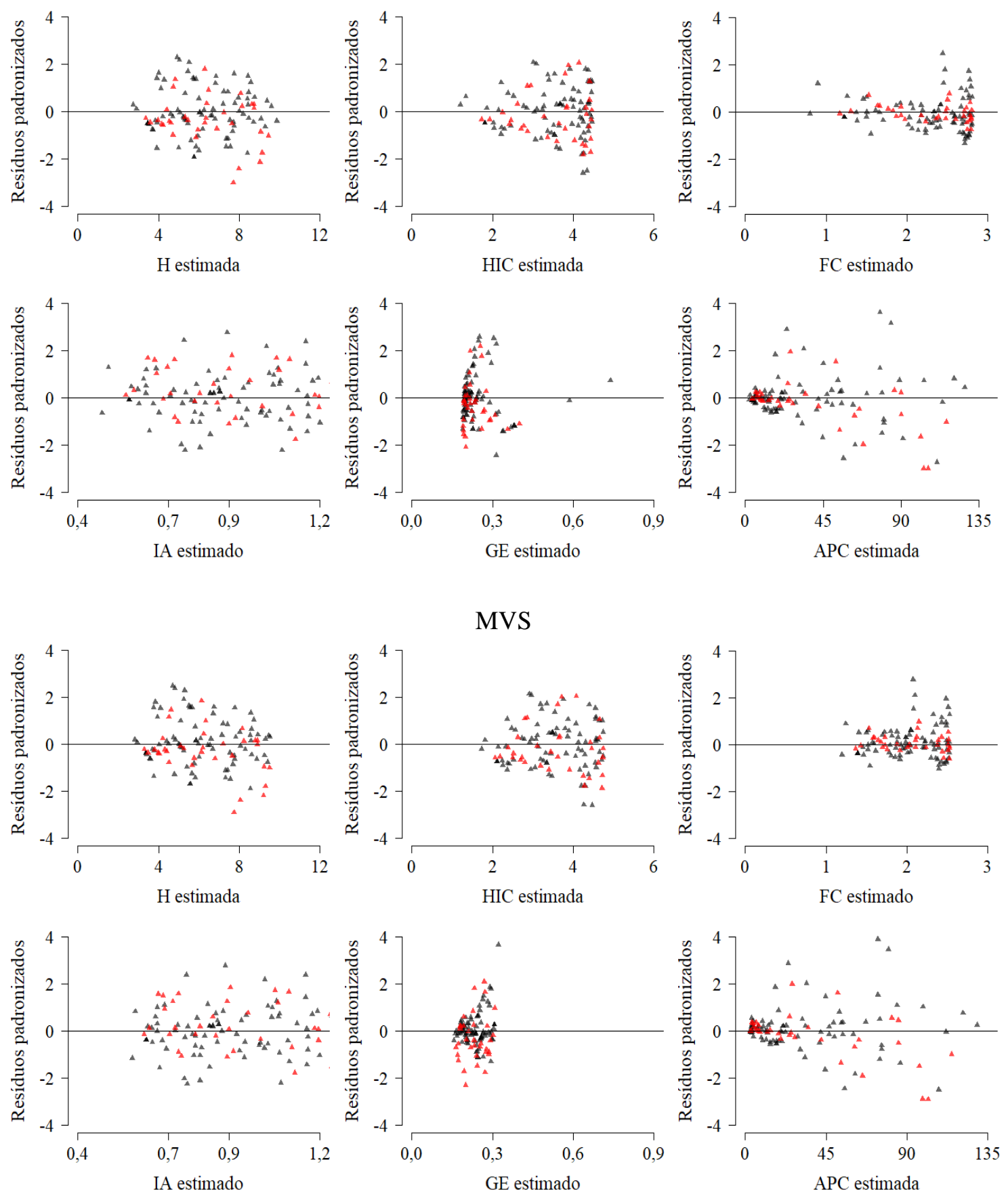

Figura 1: Resíduos padronizados gerados a partir do ajuste do modelo logístico no canto superior e máquinas de vetores de suporte com função Kernel de base radial no canto inferior. $H=$ altura total $(m)$; $H I C=$ altura do primeiro galho vivo $(m) ; F C=$ formal de copa; $I A=$ índice de abrangência $; G E=$ grau de esbeltez; $A P C=$ área de projeção de copa $\left(\mathrm{m}^{2}\right)$. Grupos de parametrização e validação em preto $e$ vermelho, respectivamente.

A maioria dos índices morfométricos cresceram de forma sigmoidal com o aumento do DAP das árvores, tendendo a uma estabilização (Figura 2). Apenas o grau de esbeltez decresceu no formato sigmoide. Mesmo com módulo do coeficiente de correlação superior a 0,7 nas fases de parametrização e validação (Tabela 1), as estimativas da equação do grau de esbeltez foram tendenciosas para árvores de maior DAP, perdendo a precisão. 

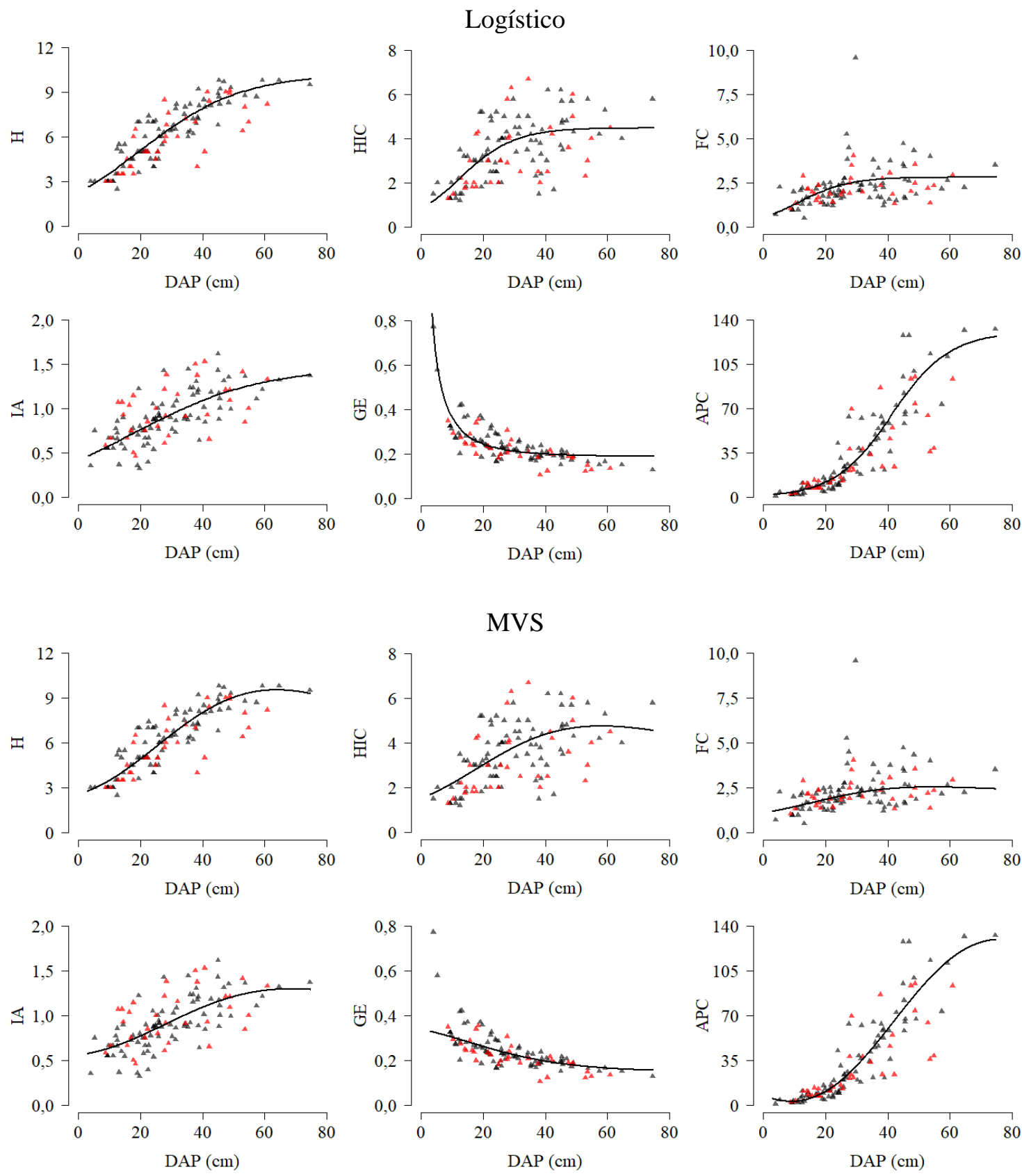

Figura 2: Valores observados e curvas geradas para as alturas e índices morfométricos da copa em função do DAP de árvores de L. tomentosa, da arborização urbana de São João Evangelista e Peçanha, em Minas Gerais, Brasil. $H=$ altura total $(\mathrm{m}) ; \mathrm{HIC}=$ altura do primeiro galho vivo $(\mathrm{m}) ; \mathrm{FC}=$ formal de copa; IA = índice de abrangência; $G E=$ grau de esbeltez; $A P C=$ área de projeção de copa $\left(\mathrm{m}^{2}\right)$. Grupos de parametrização e validação em preto e vermelho, respectivamente.

\section{DISCUSSÃO}

A análise da arborização urbana de dois municípios próximos geograficamente expandiu a amplitude de variação de DAP da L. tomentosa e contribuiu para a melhoria da qualidade preditiva dos métodos de modelagem, justificando o uso da técnica holdout de validação.

O aumento da altura total e das dimensões da copa com o acréscimo diamétrico do tronco está coerente biologicamente para árvores sem limitações espaciais de crescimento [25]. Salienta-se 
que correlações negativas de índices morfométricos da copa com o DAP devido ao contato entre copas e estabelecimento de competição podem ocorrer no âmbito de povoamentos florestais equiâneos [26]. A forte correlação $(|r| \geq 0,60)$ e negativa entre o grau de esbeltez e o DAP pode ser explicada pela própria expressão matemática do índice, que se relaciona inversamente com o diâmetro [11,22].

Em consequência da ampla variabilidade genética da produção seminal de mudas e comum indeterminação da idade de árvores no paisagismo de municípios brasileiros, a assíntota do estoque de crescimento em altura total, altura do primeiro galho vivo, formal de copa, índice de abrangência e área de projeção da copa foram estimados com precisão pela regressão logística; a qualidade preditiva desses atributos também foi confirmada visualmente. Enfatiza-se que a diminuição do grau de esbeltez à medida que aumentou o DAP é um indicativo importante de estabilidade contra a ação eólica [25, 27], reduzindo riscos de acidentes a pedestres e veículos. Apesar de o grau de esbeltez representar um indicativo de estabilidade, ainda, recomenda-se o monitoramento qualitativo e quantitativo contínuo de árvores para maior segurança e sucesso da arborização urbana.

A assíntota positiva das equações geradas evidenciou um potencial uso para delinear o comportamento biológico de troncos mais grossos. A estimativa assintótica de altura das árvores de L. tomentosa para as condições edáficas e climáticas de São João Evangelista e Peçanha é de 10,21 m, condizente com o apresentado por Lorenzi (2008) [5], que descreve uma variação de 6 a $15 \mathrm{~m}$ para espécie. Portanto, não se recomenda o seu plantio abaixo e/ou próximo de fiações elétricas aéreas. Caso seja plantada, é provável que as árvores devam ser submetidas a sucessivas podas, onerando financeiramente o projeto de arborização. Informações tradicionalmente encontradas na literatura florestal relatam variações mínimas e máximas apenas de altura e DAP, subsidiando parte do planejamento da seleção de espécies e locais adequados para a implementação de projetos paisagísticos.

A altura de inserção da copa esperada para indivíduos de maior porte da espécie em estudo é de 4,48 m, o que favorece a circulação de pedestres e pequenos veículos em segurança. A estimativa é que as árvores alcancem uma área de projeção de copa de 130,27 $\mathrm{m}^{2}$ (diâmetro de copa equivalente a 12,88 m), ou seja, 77 árvores sem limitações de crescimento forneceriam, aproximadamente, um hectare de área sombreada. Esse resultado possui relevância social e ambiental, pois o sombreamento aumenta a vida útil do asfalto, reduz a erosão e promove conforto térmico para a instalação de áreas de lazer e convivência em centros urbanos.

Diferentes formas da copa foram evidenciadas para L tomentosa, indivíduos de maior DAP, tenderam a exibir copas proporcionalmente mais largas em relação ao respectivo comprimento vertical. É provável que, na fase juvenil, árvores dessa espécie invistam mais recursos na expansão vertical da copa do que na sua largura, fato que favorece a passagem de pedestres em calçadas. Esse evento, também, pode ser comprovado na análise de tendência da relação entre diâmetro de copa e altura total, expressa pelo índice de abrangência, que tendeu ao aumento à medida que os fustes se tornaram mais grossos. Para que a copa cresça livre de impedimentos físicos, estimouse o valor médio de $6,44 \mathrm{~m}$ (raio de copa) para a distância mínima que a cova de plantio deve ficar de edificações. Logo, desde que seja adequadamente planejado, o plantio da espécie em áreas urbanas é indicado para praças, canteiros e/ou calçadões.

O emprego da função Kernel de base radial melhorou o desempenho das MVS, apresentando um ganho considerável em precisão quando comparada, principalmente, à função polinomial. Tal progresso em qualidade preditiva está de acordo com o relatado em diversas pesquisas, envolvendo o uso de funções Kernel na solução de problemas de alta complexidade [28, 29].

A eficiência computacional, simplicidade e adaptação para otimização são características que favorecem a implementação de MVS com função Kernel de base radial [19, 29]. Embora as estimativas do grau de esbeltez com aplicação dessa MVS tenham apresentado perda de precisão em fustes de menor DAP, não se observou redução acentuada de qualidade preditiva.

As curvas da Figura 2 mostraram que ambos os métodos de modelagem estabeleceram relações interdimensionais com realismo biológico, com clara tendência de acréscimo da altura total, altura do primeiro galho vivo, formal de copa, índice de abrangência e área de projeção de copa. Trata-se de um acréscimo comprovado pelos valores positivos e significativos $(p \leq 0,01)$ dos coeficientes de correlação. 
Diante da dificuldade no estabelecimento de relações interdimensionais com a copa de $L$. tomentosa, os métodos de modelagem apresentaram relativamente poucas estimativas discrepantes (resíduos padronizados fora do intervalo de -3 a 3). A qualidade preditiva dos métodos de modelagem não foi influenciada pela presença de valores atípicos.

A aplicação de MVS com função Kernel do tipo base radial para estimativa de altura e índices morfométricos apresentou desempenho preditivo similar à regressão logística. No caso específico do grau de esbeltez, a MVS modelou com maior precisão fustes mais grossos, permitindo melhor análise de tendência assintótica. Esta similaridade possui grande importância prática pois o uso das equações logísticas é simples, rápido e de fácil aplicação [12, 17]. Ressalta-se que uma constante aferição nos valores dos índices morfométricos é necessária, pois variações podem ocorrer para um mesmo genótipo em função das condições edáficas e climáticas [30, 31].

Os métodos de modelagem demonstraram capacidade em representar informações biológicas, sendo promissoras para reduzir o esforço amostral em levantamentos de copa em inventários florestais e aumentar a precisão de estimativas. Os resultados obtidos fornecem subsídios para o desenvolvimento de futuras pesquisas sobre a modelagem de relações morfométricas interdimensionais da copa de árvores. A modelagem mostrou-se uma eficiente técnica estatística capaz de representar indiretamente índices morfométricos a partir de medições rotineiras de campo, como DAP.

O sucesso da gestão da arborização urbana depende do detalhamento de informações disponíveis [1]. O interesse do setor florestal no uso de inteligência computacional [12] aliada a aplicabilidade da modelagem de relações morfométricas interdimensionais de copa contribuem para a fundamentação de decisões silviculturais e compreensão da dinâmica de crescimento vegetal em áreas urbanizadas. As limitações em se ajustar modelos não lineares ou MVS têm sido solucionadas devido a avanços tecnológicos da informática, facilitando parametrizações e a escolha da melhor alternativa de modelagem.

\section{CONCLUSÃO}

Relações morfométricas de L. tomentosa podem ser estimadas com precisão por modelos de regressão e máquinas vetor de suporte.

O modelo logístico é adequado para definição precisa da assíntota da relação do DAP com a altura total, altura do primeiro galho vivo, formal de copa, índice de abrangência e área de projeção de copa.

A técnica de máquinas vetor de suporte pode ser recomendada para predição de índices morfométricos da copa de L. tomentosa utilizando o DAP como variável preditora.

Informações como a expectativa média assintótica do estoque de crescimento de L. tomentosa em altura total, altura de inserção da copa, área de projeção da copa e raio de copa são úteis para o planejamento do local de seu plantio em áreas urbanas, indicado para praças, canteiros e/ou calçadões.

\section{REFERÊNCIAS BIBLIOGRÁFICAS}

1. Shoda T, Imanishi J, Shibata S. Growth characteristics and growth equations of the diameter at breast height using tree ring measurements of street trees in Kyoto City, Japan. Urban For Urban Green. 2020 Mar;49:126627, doi: 10.1016/j.ufug.2020.126627

2. Jones BA, Mcdermott SM. The economics of urban afforestation: insights from an integrated bioeconomic-health model. J Environ Econ Manag. 2018 Mar;89:116-135, doi: 10.1016/j.jeem.2018.03.007

3. Sartori RA, Martins GAC, Zaú AS, Brasil LSC. Urban afforestation and favela: a study in a community of Rio de Janeiro. Urban For Urban Green. 2019 Apr;40:84-92, doi: 10.1016/j.ufug.2018.10.004

4. Hilbert DR, North EA, Hauer RJ, Koeser AK, Mclean DC, Northrop R, Andreu M, Parbs S. Predicting trunk flare diameter to prevent tree damage to infrastructure. Urban For Urban Green. 2020 Mar;49: 126645, doi: 10.1016/j.ufug.2020.126645

5. Lorenzi H. Árvores brasileiras: manual de identificação e cultivo de plantas arbóreas nativas do Brasil. 5.ed. Nova Odessa: Plantarum; 2008. 384 p. 
6. Lisboa DO, Silva MA, Pinho DB, Pereira OL, Furtado GQ. Diversity of pathogenic and endophytic Colletotrichum isolates from Licania tomentosa in Brazil. For Pathol. 2018 May;48:1-11, doi: 10.1111/efp. 12448

7. Teixeira LL, Silva GA, Macedo JBM, Almeida MG, Moura MFV. Physicochemical characterization and phenolic profile of oiti fruits (Licania tomentosa Benth Fristch). J Nutr Food Technol. 2019;2(1):712, doi: 10.30881/jnfrt.00015

8. Canetti A, Mattos PP, Braz, EM, Pellico Netto S. Life pattern of urban trees: a growth-modelling approach. Urban Ecosyst. 2017 Mar;20:1057-1068, doi: 10.1007/s11252-017-0659-0

9. Hofman J, Bartholomeus H, Janssen S, Calders K, Wuyts K, Wittenberghe S, Samson R. Influence of tree crown characteristics on the local PM10 distribution inside an urban street canyon in Antwerp (Belgium): a model and experimental approach. Urban For Urban Green. 2016 Dec;20:265-276, doi: 10.1016/j.ufug.2016.09.013

10. Seidel D, Ruzicka K, Puettmann K. Canopy gaps the shade of Douglas-fir crowns in the western Cascades, Oregon. Foreco. 2016 Mar;363:31-38, 2016. doi: 10.1016/j.foreco.2015.12.024

11. Sterba, H. Forstliche Ertragslehre. Wien: Universität für Bodenkultur, 1991. 160 p.

12. Campos JCC, Leite HG. Mensuração Florestal: perguntas e respostas. 5. ed. Viçosa: Ed. UFV; 2017. $636 \mathrm{p}$.

13. Silva, GCS, Calegario N, Silva AAL, Cruz JP, Leite HG. Site index curves in thinned and non-thinned eucalyptus stands. Foreco. 2018 Jan;408:36-44, doi: 10.1016/j.foreco.2017.10.036

14. Vieira GC, Mendonça AR, Silva GF, Zanetti SS, Silva MM, Santos AR. Prognoses of diameter and height of trees of eucalyptus using artificial intelligence. Sci Total Environ. 2018 Apr;619-620:14731481, doi: 10.1016/j.scitotenv.2017.11.138

15. Luo J, Zhang M, Zhou X, Chen J, Tian Y. Tree height and DBH growth model establishment of main tree species in Wuling Mountain small watershed. Environ Earth Sci. 2018,108(4):1-6, doi: 10.1088/1755-1315/108/4/042003

16. Melo EA, Calegario N, Mendonça AR, Possato EL, Alves JA, Isaac Júnior MA. Modelagem não linear da relação hipsométrica e do crescimento das árvores dominantes e codominantes de Eucalyptus sp. Ciênc Florest. 2017 Out-Dez;27(4):1325-1338, doi: 10.5902/1980509829895

17. Tjørve KMC, Tjørve E. A proposed family of unified models for sigmoidal growth. Ecol Model. 2016 Set;359:117-127, doi: 10.1016/j.ecolmodel.2017.05.008

18. Ukalska J, Jastrzębowski S. Sigmoid growth curves, a new approach to study the dynamics of the epicotyl emergence of oak. Folia For Pol. 2019;61(1):30-41, doi: 10.2478 / ffp-2019-0003

19. Vapnik VN. The nature of statistical learning theory. New York: Springer-Verlag, 1995. 188 p.

20. Kumar VA, Souza D, Lindén R, Laakson M. Prediction of student final exam performance in an introductory programming course: development and validation of the use of a support vector machineregression model. Asian Educ e-Learn. 2019 Feb;7(1):2321-2454, doi: 10.24203/ajeel.v7i1.5679

21. Herceg S, Andrijic ZU, Bolf N. Development of soft sensors for isomerization process based on support vector machine regression and dynamic polynomial models. Chem Eng Res Des. 2019 Sep;149:95-103, doi: 10.1016/j.cherd.2019.06.034

22. Durlo MA, Denardi L. Morfometria de Cabralea canjerana, em mata secundária nativa do Rio Grande do Sul. Ciênc Florest. 1998;8(1):55-66, 1998, doi: 10.5902/19805098351

23. Meyer D, Dimitriadou E, Hornik K, Weingessel A, Leisch F, Chang C, Lin C. e1071: Misc Functions of the Department of Statistics, Probability Theory Group (Formerly: E1071), TU Wien. R package version 1.6-8, 2017.

24. R Core Team. R: A language and environment for statistical computing. Vienna: R Foundation for Statistical Computing. 2018.

25. Costa EA, Finger CAG. Efeito da competição nas relações dimensionais de Araucária. Floram. 2017 Nov;24:e20150145, doi: 10.1590/2179-8087.014515

26. Trindade RNR, Lafetá BO, Aguiar VF, Silva AG, Ferraro AC, Penido TMA, Vieira DS. Morfometria da copa de povoamento de Eucalyptus grandis Hill ex Maiden x E. urophylla S. T. Blake em diferentes espaçamentos de plantio. Sci Forest. 2019 Mar;47(121):83-91, doi: 10.18671/scifor.v47n121.08

27. Costa EA, Finger CAG, Fleig FD. Influência da posição social nas relações morfométricas de Araucaria angustifolia, Ciênc Florest. 2016 Jan-Mar;26(1):225-234, doi: 10.5902/1980509821116

28. Ghitescu R, Curteanu S, Mihailescu C, Volf I, Leon F, Gilca AI, Popa VI. Support vector machine combined with genetic algorithm for optimization of microwave-assisted extraction of polyphenols from spruce wood bark. Cellulose Chem Technol. 2017;51(3-4):203-213.

29. Silva MBP, Escobedo JF, Santos CM, Rossi TJ, Silva SHMG. Performance of the Angstrom-Prescott Model (A-P) and SVM and ANN techniques to estimate the daily global solar irradiation in Botucatu/SP/Brazil. J Atmos Sol-Terr Phy. 2017 Jul;160:11-23, doi: 10.1016 / j.jastp.2017.04.001 
30. Coble AP, Autio A, Cavalieri MA, Binkley D, Ryan MG. Converging patterns of vertical variability in leaf morphology and nitrogen across seven Eucalyptus plantations in Brazil and Hawaii, USA. Trees. 2014 Aug;28:1-15, doi: 10.1007/s00468-013-0925-6

31. Sapijanskas J, Paquette A, Potvin C, Kunert N, Loreau M. Tropical tree diversity enhances light capture through crown plasticity and spatial and temporal niche differences. Ecology. 2014 Sep;25(9):24792492, doi: $10.1890 / 13-1366.1$ 